\title{
A Collection of Challenging Optimization Problems in Science, Engineering and Economics
}

\author{
Dhagash Mehta \\ Dept of Applied and Computational Mathematics and Statistics \\ University of Notre Dame \\ Notre Dame, IN 46556, USA, and \\ Centre for the Subatomic Structure of Matter \\ Department of Physics, School of Physical Sciences \\ University of Adelaide, Adelaide, \\ South Australia 5005, Australia. \\ Email: dmehta@nd.edu
}

The University of Adelaide Preprint No.

ADP-15-9/T911

\begin{abstract}
Function optimization and finding simultaneous solutions of a system of nonlinear equations (SNE) are two closely related and important optimization problems. However, unlike in the case of function optimization in which one is required to find the global minimum and sometimes local minima, a database of challenging SNEs where one is required to find stationary points (extrama and saddle points) is not readily available. In this article, we initiate building such a database of important SNE (which also includes related function optimization problems), arising from Science, Engineering and Economics. After providing a short review of the most commonly used mathematical and computational approaches to find solutions of such systems, we provide a preliminary list of challenging problems by writing the Mathematical formulation down, briefly explaning the origin and importance of the problem and giving a short account on the currently known results, for each of the problems. We anticipate that this database will not only help benchmarking novel numerical methods for solving SNEs and function optimization problems but also will help advancing the corresponding research areas.
\end{abstract}

\section{INTRODUCTION}

Development of methods for local and global optimization, which include finding the global minimum, local minima and saddle points, of nonlinear multivariate objective functions, say $F(\mathbf{x})$, has always been one of the most active areas of research in Mathematics and Computer Sciences, due to their applications in many areas of Science, Engineering, Economics, etc. Here, $F(\mathrm{x})$ is usually a real-valued function from $\mathbb{R}^{N}$ to $\mathbb{R}$. The $N$-dimensional space is made of the degrees of freedom of the physical system.

\author{
Crina Grosan \\ Department of Computer Science \\ Brunel University, London, UK \\ Email: crina.grosan@brunel.ac.uk
}

The most general form of the optimization problems is to find the stationary points (SPs) of $F(\mathbf{x})$, defined as the simultaneous solutions of the system of equations $f_{i}(\mathbf{x})=\partial F(\mathbf{x}) / \partial x_{i}=0$, for all $i=1, \ldots, N$. The SPs at which exactly $i$ eigenvalues of the Hessian are negative definite, and the remaining $N-i$ eigenvalues are positive definite, are called saddles of index $i$, with $i=0$ SPs also known as the minima of $F(\mathbf{x})$. The SP at which $F(\mathbf{x})$ attains its lowest value is known as the global minimum, provided $F(\mathbf{x})$ is bounded from below. The SPs with at least one 0 eigenvalue are called singular SPs.

As is common to most nonlinear problems, an analytic calculation of the SPs is extremely difficult, and in most cases impossible. Hence, one has to rely on computational methods. Leaving the certification of numerical solutions aside [1]-[4], the Newton-Raphson (NR) approach has been a popular method to find SPs of nonlinear functions. There, one refines an initial guess through successive iterations and hopes to converge to a solution. However, the NR method may often converge slowly, or even worse but not uncommon, may diverge depending on the initial guess, and may behave erratically near singular solutions [5], [6].

One can also resort to alternative methods such as the gradient-square minimization method: instead of solving $f_{i}(\mathbf{x})=0$ directly, here one minimizes the sum of squares $W=\sum_{i=1}^{N} f_{i}(\mathbf{x})^{2}$ using traditional numerical methods, such as conjugate gradient [7], [8]. When imposing the further constraint $W=0$, the corresponding minima of $W$ are then the desired solutions of $f_{i}(\mathbf{x})=\mathbf{0}$, for all $i=1, \ldots, N$. However, the number of minima with $W>0$, which are not the solutions of $f_{i}(\mathbf{x})$, generally outweighs the desired minima at which $W=0$. Moroever, these non-solutions 
may also be singular making the minimization problem ill-conditioned [9], [10]. Hence, this approach turns out to be very inefficient in practice [9], [11]. Instead, a biased gradient squared descent framework [12] may provide a more useful alternative to the gradient squared minimization method.

There are other systematic approaches such as the one based on the Broyden-Fletcher-Goldfarb-Shanno (BFGS) algorithm [13], [14], or eigenvector-following [9], [11] (implemented in the OPTIM package, which also includes many other geometry optimization techniques, such as a modified version of the limitedmemory BFGS algorithm [13], [14], single- and doubleended [15]-[20] searches).

For SNEs having polynomial-like nonlinearity, symbolic algebraic geometry methods based on the Gröbner basis technique can guarantee to find all the complex solutions [21]. However, the algorithms are known to have exponential space complexity, which may make solving even a moderately sized SNE prohibitively difficult. Another rigorous approach which can guarantee to find all the solutions of a system of nonlinear equations is an interval based method [22]. However, this approach has only proved successful for a very small systems and SPs so far because it is based on bisections of the ranges and the computation blows up by increasing the number of variables. In the homotopy continuation approach [23], on the other hand, one starts with a new SNE that is qualitatively similar to the SNE to be solved, and whose solutions are known or can be easily obtained. Then, each solution of the new system is homotopically continued to eventually obtain a solution of the system to be solved. For a general nonlinear system, this method does not usually guarantee to find all solutions 1 .

Recently, a specialized homotopy continuation method based on algebraic geometry, namely the numerical polynomial homotopy continuation (NPHC) method [26]-[28], has captured the attention due to its ability of finding all the isolated SPs of $F(\mathbf{x})$ having polynomiallike nonlinearity. Several good implementations of the NPHC method are now available[27], [29]-[31] and the method is applied to many different areas in Science and Engineering in recent years [32]-[44]. In this method, after coming up with an upper bound on the number of isolated complex solutions of the given SNE, the system is continuously deformed from a different system whose solution count agrees with the upper bound to finally obtain all the complex solutions of the original system. In the end, only real solutions are retained being physically relevant. However, in many real life applications, the number of complex solutions may be extremely large making the task of computing all of the real solutions

\footnotetext{
${ }^{1}$ There have been attempts to construct specialized homotopies which guarantees to find all isolated solutions for general (i.e., nonpolynomial) SNE (see, e.g.,[24], [25]), though these claims have not yet been rigorously proven.
}

a prohibitively difficult task.

An inversion-relaxation method [45] was also recently introduced in which, to obtain stationary points of a given $F(\mathbf{x})$ which is bounded from above and below, one relaxes from a solution of index $N$ to find all the saddles of index $(N-1)$ that are connected to the maximum. Relaxing from each of these saddles of $(N-1)$ index, one then obtains all the saddles of $(N-2)$ index that are connected to the corresponding saddle of $(N-1)$ index, and so on. One can then obtain many saddles of all the possible indices starting from one maximum. If all the maxima of $F(\mathbf{x})$ are known, then all the stationary points may be found using this method. Further investigations in addition to a rigorous proof of the previous statement is still needed.

In recent years, soft computing methods (especially population based methods) prove to be efficient in finding multiple solutions with relatively less information about the system (i.e. without using derivatives, etc.). But there are still challenges for these methods, particularly in the following aspects: dealing with systems which have a large number of equations; finding a large number of distinct solutions; and scaling an efficient method for a simpler system to larger systems. The work in [46] is among the first approaches which transform a system of equations into a multiobjective optimization problem. Although various ways to transform a system of equations into an optimization problem have been proposed [47]-[57], none of these methods can detect all the solutions, and are biased for problems in specific areas. The main ways in which population based heuristics transform a system of equations into an optimization problem are single-objective optimization based methods, constrained optimization based methods, and multiobjective optimization based methods.

However, as with many other Mathematical areas, optimization method development is mostly disconnected to the real life applications. Most of the conventional test systems [58] currently used by the optimization community, though while serving the purpose of benchmarking the novel methods may not appeal the Scientific community. Another reason for the disjointedness is the different terminology, related to the corresponding scientific application, being used among the scientific communities for the same underlying Mathematics.

Our goal in this paper is to bridge the gap by collecting some of the challenging systems of equations as optimization problems arising from real life applications in the terminology which is accessible to the optimization and computational mathematics community. Hence, our list of challenging systems may not only provide motivation and benchmarking for novel algorithms, but solving them for the yet unsolved cases will provide advancement in the respective areas the systems arise from. The models we provide are described generic, given by their general representation. They can be used 
as benchmarks of variable size, some being simpler (lower dimension) than the others. All the systems have multiple solutions, in some cases this increases exponentially with the dimensionality of the system. Finding all solutions will be one of the main challenges of the population based algorithms. As such a set of benchmarks is currently missing in the evolutionary computation literature, we believe that our work herein will be of help for all the researchers working on computational approaches (especially in evolutionary computation field) for solving complex systems of equations.

In the remainder of this paper, we provide a brief introduction, Mathematical formulation, and a list of open Mathematical problems for each of the optimization models We do not however intend to provide a complete list of references nor a complete list of available results for each of the problems. Rather we refer the reader to the corresponding databases, if available.

\section{Challenging Problems}

In this Section, we list out a few of the challenging problems arising in various research areas.

\section{A. Chemical and Physical Clusters}

In theoretical chemistry, physics and many other areas in Science and Engineering, exploring the hypersurface defined by a multivariate function, $V(\mathbf{x})$, called the potential energy function, plays a very important role in understanding and describing the physics and chemistry of the phenomenon. The hypersurface is called the potential energy landscape (PEL).

In fact, a variety of methods based on the SPs have recently attraced a lot of attention of both chemists and physicists, due to their applications to many-body systems as diverse as metallic clusters, biomolecules, structural glass formers, and coarse-grained models of soft matter, etc. [59], [60]. Finding SPs of $V(\mathbf{x})$ provides the foundations for global optimization [61]- 63], thermodynamic sampling to overcome broken ergodicity [64]-[67], as well as rare event dynamics [68]-[72] within the general framework of PEL theory [59]. Below, we list out a few important potentials coming from chemistry and physics applications as optimization problems.

1) The Nearest-neighbour $\phi^{4}$ Model: The twodimensional nearest-neighbor $\phi^{4}$ model has been widely studied because (1) it is one of the simplest models with a continuous configuration space, (2) it exhibits a phase transition in the same universality class as the twodimensional Ising model. For an $N \in \mathbb{Z}^{+}$and $J, \lambda, \mu \in$ $\mathbb{R}$ the model, in $N^{2}$ variables $\mathbf{x}=\left(x_{11}, x_{12}, \ldots, x_{N N}\right)$, is $V(\mathbf{x})$ given by

$V(\mathbf{x})=\sum_{(i, j) \in \Lambda}\left(\frac{\lambda}{4 !} x_{i j}^{4}-\frac{\mu^{2}}{2} x_{i j}^{2}+\frac{J}{4} \sum_{(k, l) \in \mathcal{N}_{(i, j)}}\left(x_{i j}-x_{k l}\right)^{2}\right)$

where $\Lambda \subset \mathbb{Z}^{2}$ is the standard square lattice with $N^{2}$ lattice-sites and $\mathcal{N}_{(i, j)} \subset \Lambda$ is the four nearest-neighbor sites of $(i, j)$. The $N^{2}$ stationary equations are given by

$\frac{\partial V(\mathbf{x})}{\partial x_{i j}}=\frac{\lambda}{3 !} x_{i j}^{3}+\left(4 J-\mu^{2}\right) x_{i j}-\sum_{(k, l) \in \mathcal{N}_{(i, j)}} J x_{k l}=0$

for each pair of $i, j=1, \ldots, N$. The traditional boundary conditions is the periodic one, $\lambda=3 / 5$ and $\mu^{2}=2$. Only real solutions are physical for this model.

The model has played a crucial role in studying link between the topology of the potential energy landscape with the phase transition [35], [39], [73]. Moreover, the model shows an interesting behavour while varying the parameter $J$ from 0 to 1 , i.e., one can go from the case when all the solutions are real to only an extremely small fraction are real. A variety of computational methods have been used to explore the PEL of this model.

The NPHC method has found all the SPs for $N=$ 3,4 in a previous study [35], [74]. However, this model poses a particularly tough challenge to the method since the total number of solutions in $\mathbb{C}^{N^{2}}$, counting multiplicity, is always equal to its total degree (the Bezout bound) $3^{N^{2}}$, which grows quickly as $N$ increases. For example, for $N=6$ and 7 , the total degree exceeds $10^{17}$ and $10^{23}$, respectively. Hence, finding all complex solutions is clearly unfeasible with current technology for large values of $N$. More recently, the Newton homotopy method has been employed to find many real solutions of this model for larger $N$, though without a guarantee of finding all the real solutions [6]. Thus, this model still needs to be studied with more refined methods. On the other hand, due to the simplicity of the model, it can be used as a benchmark model.

2) The Thomson Problem: The Thomson problem [75] is to find the minimum energy of the system made of $N$ electrons restricted to move on the surface of a sphere of unit radius. The model was originally proposed by Sir JJ Thomson as a natural consequence of his atomic model called the plum-pudding model. Though the model turned out not to be a correct model for the atoms due to experimental evidence, it has turned out to be very interesting model in chemistry, physics and mathematics. The global minima found by numerical methods have been observed to be geometrically irregular: though the global minima for $N=4,6$ and 12 are the expected platonic solids, surprisingly, those for $N=8$ and 20 are not platonic solids. The Thomson problem and its generalizations have also been used to model clusters of proteins on a shell, colloid particles, 
fullerene patterns of carbon atoms, etc. In addition, the model has got the status of a standard benchmark system for any new optimization routines. Finding the exact global minimum of the model has also been an active area of research, though the exact results are known only for a handful of $N \mathrm{~s}(N=2-6$ and 12) [76], [77].

Because the electrons interact with each other with the Coulomb potential, the potential energy function of this system is given by

$$
V_{\mathrm{Th}}(\vec{x})=\sum_{1 \leq i<j \leq N} \frac{1}{r_{i j}}
$$

where $r_{i j}=\sqrt{\left(x_{i}-x_{j}\right)^{2}+\left(y_{i}-y_{j}\right)^{2}+\left(z_{i}-z_{j}\right)^{2}}$, with constraints $x_{i}^{2}+y_{i}^{2}+z_{i}^{2}=1$ for all $i=1, \ldots, N$. To remove the rotational symmetry of the system, we fix $x_{1}=y_{1}=y_{2}=0$ and $z_{1}=1$.

The model has gained special attention recently as it appeared as the 7th problem in Steven Smale's list of eighteen unsolved problems for the 21 st century [78]. This problem was indeed motivated by finding a good starting polynomial for a homotopy algorithm for realizing the Fundamental Theorem of Algebra. For the sepcial case of finding the global minimum of the model, in Ref. [79] it was shown that good initial points to find the global minimum of the Thomson model are in fact complex roots (projected to 2-sphere) of random univariate polynomials.

3) Lennard-Jones Clusters: One of the most popular model for atomic interactions, in theoretical chemistry is the Lennard-Jones potential [80], which is defined as

$$
V_{\mathrm{LJ}}(\vec{x})=4 \epsilon \sum_{i=1}^{N} \sum_{j=i+1}^{N}\left[\left(\frac{\sigma}{r_{i j}}\right)^{12}-\left(\frac{\sigma}{r_{i j}}\right)^{6}\right],
$$

where $\epsilon$ is the pair well depth, and $2^{1 / 6} \sigma$ is the equilibrium pair separation. We take $\epsilon=\sigma=$ 1. Here, same as in the Thomson problem, $r_{i j}=$ $\sqrt{\left(x_{i}-x_{j}\right)^{2}+\left(y_{i}-y_{j}\right)^{2}+\left(z_{i}-z_{j}\right)^{2}}$ is the distance between atoms $i$ and $j$. To remove the global degrees of freedom of the model coming via the rotational and translational invariance of the system, we can fix $x_{1}=y_{1}=z_{1}=y_{2}=z_{2}=z_{3}=0$. Thus, in total there are $3 N-6$ variables in $V_{\mathrm{LJ}}$ yielding $3 N-6$ equations $\nabla V_{\mathrm{LJ}}=\mathbf{0}$. The model has gained popularity among the theoretical and computational chemists because it is simple enough to perform molecular dynamics simulations and also because it is fairly accurate approximation of the actual atomic interactions validated by experiemental observations. The minima of this model represent individual molecular configurations. There is a huge literature available addressing the global and local optimization issues of the PEL of this model. An extensive search for minima and saddle points has been carried out in [9] for $N$ up to 13, and a search for minima and saddles of index one (transition states) for $N=14$ was presented in [81]. Recently, in Ref. [82], one of the authors has worked out a Newton homotopy method which can not only efficiently find multiple stationary points of this model, but also can find singular solutions of the model. The latter task has been proved to be prohibitively difficult using traditional methods in which one has to invert the hessian matrix at such singular solutions.

4) Morse Clusters: The Morse potential [83] is given as

$$
V(r)=\epsilon \sum_{i<j}\left(e^{\rho\left(1-\frac{r_{i j}}{r_{e}}\right)}\left(e^{\rho\left(1-\frac{r_{i j}}{r_{e}}\right)}-2\right)\right),
$$

where $r_{i j}$ is again the distance between atoms $i$ and $j, \epsilon$ is the dimer well depth and $r_{e}$ equilibrium bond length. Since these two parameters do not affect the geometry of the PEL, we can conveniently set them to be unity. We can again fix 6 of the coordinates to remove the rotational and translational symmetries of the system to have eventually a system of $3 N-6$ equations and $3 N-6$ variables.

The most important parameter in this model is $\rho$ which is dimensionless. $\rho$ determines the range of the inter-particle forces: low values of $\rho$ correspond to long range interactions because increases the range of the attractive part of the potential and softens the repulsive wall, thus widening the potential well. Similarly, large values correspond to short range interactions. The ability to continuously varying the interaction range of the particles has made this model widely popular to study a range of chemical phenomena from intermolecular potential of $C_{60}$ to alkali metals. In fact, at $\rho=6$, the above potential has the same curvature at the bottom of the well as the Lennard-Jones potential. A database of the known minima and transition states of this model for various values of $\rho$ are available at the Cambridge Energy Landscape database http: / / www-wales.ch. cam.ac.uk/CCD.html.

5) The $X Y$ Model: The $X Y$ model is one of the simplest potentials with continuous degrees of freedom (unlike the Ising model in which the configuration space is discrete) in theoretical physics and chemistry, though its potential energy landscape is very rich and interesting, and has been helpful in understanding general features of potential energy landscapes.

The function to be extremized for the $X Y$ model on a $d$-dimensional cubic lattices $\Lambda$ of side length $L$ is

$$
H=\frac{1}{2} \sum_{k \in \Lambda} \sum_{l \in \mathcal{N}(k)}\left[1-J_{k, l} \cos \left(\theta_{k}-\theta_{l}\right)\right] .
$$

Here, the total number of lattice sites is $N=L^{d}$, and for each lattice site $k \in \Lambda$ the angular variable $\theta_{k} \in(-\pi, \pi] . \mathcal{N}(k)$ denotes the set of nearest-neighbors of lattice site $k$. Moreover, the parameters $J_{k, l}$ are the random disorders which are i. i. d. picked from some random distribution. One has to pick a boundary condition here due to the nearest-neighbour terms, the 
usual choices being periodic and anti-periodic boundary conditions. For the periodic boundary conditions, to remove the global $\mathrm{O}(2)$ symmetry, exactly one of the angles is fixed to zero.

The function (6) also appears in many other areas such as in statistical physics [84], complex systems [85], lattice field theories [32], [86], [87], etc. The model is used to model low-temperature superconductivity, superfluid helium, hexatic liquid crystals, and other phenomena.

For the one-dimensional case, all SPs of this model are analytically found in [32], [87]-[89]. Using the SPs of the one-dimensional model, a class of SPs for the two- and three-dimensional cases can be constructed [90] (see also [38], [91]). For the two-dimensional case, for small number of lattice-sites, all the SPs were found using the NPHC method in [32], [38], [87], [92]. Using other traditional numerical methods [32], [90], it was then shown that the number of isolated SPs, as well as the number of minima, of the $X Y$ model in two- and three-dimensions increases exponentially as $N$ increases. Similar results were obtained from the Kuramoto model point of view in [93]. It was also shown that even after removing the global $O(2)$ symmetry, the model possess many continuous SPs. Several attempts for finding the global minimum of this model have also been made, e.g., using Simulated Annealing in [94]. However, the model has proven to be a very challenging optimization problem and even for moderate $N$ many features of the PEL is yet to be explored.

\section{B. Polynomial systems in Economics}

The computation of equilibria in economics leads to systems of polynomial equations. Also known as pseudo-games, social equilibrium problem, equilibrium programming, coupled constrained equilibrium problem, abstract economy [95]. Using the notations and definitions from [96], the $n$-person game is defined by $n$ players, labelled $1,2, \ldots, n$. Each player $i$ has $d_{i}$ pure strategies labeled $1,2, \ldots, d_{i}$. Each player has an objective function that depends both on his own strategies and the strategies of the other players. This function is called utility function or payoff function. The game is defined by $n$ payoff matrices $X^{(1)}, X^{(2)}, \ldots, X^{(n)}$, one for each player. Each matrix $X^{(i)}$ is an n-dimensional matrix of format $d 1 \times d 2 \times \ldots \times d n$ whose elements are rational numbers. The element $X_{j_{1} j_{2} \ldots j_{n}}^{(i)}$ represents the payoff for player $i$ if the other players $1,2, \ldots, n$ select strategies $j_{1}, j_{2}, \ldots, j_{n}$ respectively. Each player selects a mixed strategy given by $p^{(i)}=\left(p_{1}^{(i)}, p_{2}^{(i)}, \ldots, p_{d_{i}}^{(i)}\right)$, where $p_{j}^{(i)}$ is the probability of player $i$ to select strategy $j$. The vector $p^{(i)}$ is a probability distribution of player $i$ on his set of pure strategies. $X^{(i)}$

$$
\pi_{i}=\sum_{j_{1}=1}^{d_{1}} \sum_{j_{2}=1}^{d_{2}} \ldots \sum_{j_{n}=1}^{d_{n}} X_{j_{1} j_{2} \ldots j_{n}}^{(i)} \times p_{j_{1}}^{(1)} p_{j_{2}}^{(2)} \ldots p_{j_{n}}^{(n)}
$$

Since the variables of the problem are probabilities, we have that $p_{j}^{(i)} \geq 0, \forall i, j$, and $p_{1}^{(i)}+p_{2}^{(i)}++p_{d_{i}}^{(i)}=$ $1, \forall i$ which means that $p=\left(p^{(i)}\right)$ is a point in the product of simplices $\triangle=\triangle d_{1}-1 \times \triangle d_{2}-1 \times \ldots \times$ $\triangle d_{n}-1$.

A point $p \in \triangle$ is a Nash equilibrium if none of the players can increase his payoff by changing his strategy while the other $n-1$ players keep their strategies unchanged. This can be expressed as a system of polynomial constraints in the variable vectors $p \in \triangle$ and $\pi=\left(\pi_{1}, \ldots, \pi_{n}\right) \in \Re^{n}$, with the following multilinear polynomial for each $p_{k}^{(i)}$ :

$$
\begin{aligned}
p_{k}^{(i)}\left(\pi_{i}-\sum_{j_{1}=1}^{d_{1}} \ldots\right. & \sum_{j_{i-1}=1}^{d_{i-1}} \sum_{j_{i+1}=1}^{d_{i+1}} \cdots \sum_{j_{n}=1}^{d_{n}} X_{j_{1} j_{2} \ldots j_{n}}^{(i)} \\
& \left.\times p_{j_{1}}^{(1)} \ldots p_{j_{i-1}}^{(i-1)} \times p_{j_{i+1}}^{(i+1)} \ldots p_{j_{n}}^{(n)}\right)
\end{aligned}
$$

which, together with the constraints, represents a system of $n+d_{1}+\ldots+d_{n}$ equations in $n+d_{1}+\ldots+d_{n}$ variable. Each polynomial is the product of a linear polynomial and a multilinear polynomial of degree $n-1$.

A solution $(p, \pi) \in \triangle \times \Re^{n}$ represents a Nash equilibrium for the game if and only if $(p, \pi)$ is a zero of the polynomials in (7) and each expression in the parenthesis is nonnegative.

\section{Edge matching puzzles}

Edge-matching problems are popular puzzles in which, given a set of pieces and a grid, the goal is to place the pieces on the grid such that the edges of the connected pieces match. Edge-matching puzzles are challenging because there is no global image as guidance and there is no guarantee that two pieces fitting together are in the right positions. Edge-matching problems are proved to be NP-complete [97]. A direct implication and practical application of the edge matching puzzles is in image reconstruction.

The work in [98] formulates the edge matching problem as a systems of polynomial equations derived from the pieces of the puzzle. Solutions of the system represent solutions of the puzzle. The authors consider a particular instance of the problem in which a set of pieces of known shapes and edge colours. is given. The puzzle is bounded by a frame and each edge must match either an edge of another piece or the frame. The puzzle has $\mathrm{N}$ pieces and the puzzles as considered as a 2dimensional polygons. Each piece $i$ of the puzzle is represented by its location $t_{i} \in \Re^{2}$ given by its center and its set $E_{i}$ of edges. Each edge $j \in E_{i}$ is described by the relative location of its center $b_{i, j}$ with respect to the piece center, its color $c_{i, j}$ and its orientation or inclination given by the angle $\theta_{i, j}$. The absolute 
location of the $j$ th edge of the $i$ th piece is given by the sum $t_{i}+b_{i, j}$.

The pieces corresponding to the puzzle frame have $i$ $=0$ and the properties of its edge elements $b_{0, j}, c_{0, j}$ and $\theta_{0, j}$. The only operation that can be applied to puzzle's pieces is translation. The goal of the game is to find a translation $\left(t_{1}, \ldots, t_{N}\right)$ such as all edge elements pair with matching edge elements in their spatial location, colour and orientation.

If $\left(t_{1}, \ldots, t_{N}\right)$ is a solution of the puzzle then it is also a solution for the system of equations given by:

$$
\sum_{i, j} s_{i, j}(c, \theta) f\left(t_{i}+b_{i, j}\right)=0
$$

for every $(c, \theta)$ and every real valued function $f: \Re^{2} \rightarrow$ $\Re$, where $s_{i, j}(c, \theta)$ is a signed indicator function w.r.t. $(c, \theta)$ and is given by:

$$
s_{i, j}(c, \theta)= \begin{cases}1, & c_{i, j}=c, \quad \theta_{i, j}=\theta \\ -1, & c_{i, j}=c, \quad \theta_{i, j}=\theta+\pi \\ 0, & \text { otherwise }\end{cases}
$$

Different choices of $f$-function are possible. There trivial one reduces the system to:

$$
\sum_{i, j} s_{i, j}(c, \theta)\left(t_{i}+b_{i, j}\right)=0
$$

The fact is that the converse of this statement does not hold and not every solution of the system is a solution of the puzzle. In order to have the converse valid as well, consider $f$ as an exponential function defined as (for a given $k \in \Re^{2}$ ) $f_{k}(u)=e^{k^{T} u}$. In this case the equation (8) becomes:

$$
\sum_{i, j} s_{i, j}(c, \theta) e^{k^{T}\left(t_{i}+b_{i, j}\right)}=0
$$

which by replacing $T_{i}^{k}=\left(e^{t_{i}}\right)^{k}=e^{k^{T_{t_{i}}}}$

$$
\sum_{i, j} s_{i, j}(c, \theta) T_{i}^{k}=0
$$

This leads to the conclusion that $t_{1}, \ldots, t_{N}$ is identified with $T_{1}, \ldots, T_{N}$ and the fact that the converse holds. This, a solution of the system of equation is a solution of the puzzle.

\section{CONCLUSION}

We presented several models which compose complex systems of equations, together with their description. These models arrive in a variety of scientific areas and are of great importance. The equations are presented in a general format so that those interested in using them as benchmarks can build their own instance of the system. The scope of this work is to offer the research community (especially the computational science community) a common set of difficult equations systems benchmarks. The systems presented are difficult both in terms of number of equations they contain as well as in number of alternative solutions. As such a repository is mandatory for testing the performance of the newly proposed methods for solving systems of equations, we believe that the work here represents a starting point and that more system models will be considered in the future.

\section{ACKNOWLEDGMENT}

DM was supported by a DARPA Young Faculty Award and an Australian Research Council DECRA fellowship.

\section{REFERENCES}

[1] J. D. Hauenstein and F. Sottile. alphaCertified: certifying solutions to polynomial systems. 2010.

[2] J. Cleveland, J. Dzugan, J. D. Hauenstein, I. Haywood, D. Mehta, A. Morse, L. Robol, and T. Schlenk. Certified counting of roots of random univariate polynomials. ArXiv e-prints, December 2014.

[3] Dhagash Mehta, Jonathan D. Hauenstein, and David J. Wales. Communication: Certifying the potential energy landscape. The Journal of Chemical Physics, 138(17):-, 2013.

[4] Dhagash Mehta, Jonathan D. Hauenstein, and David J. Wales. Certification and the potential energy landscape. The Journal of Chemical Physics, 140(22):-, 2014.

[5] A. Griewank and M. R. Osborne. Analysis of newton's method at irregular singularities. SIAM Journal on Numerical Analysis, 20(4):747-773, 1983.

[6] Dhagash Mehta, Tianran Chen, Jonathan D. Hauenstein, and David J. Wales. Communication: Newton homotopies for sampling stationary points of potential energy landscapes. The Journal of Chemical Physics, 141(12):-, 2014.

[7] L Angelani, R Di Leonardo, G Ruocco, A Scala, and F Sciortino. Saddles in the energy landscape probed by supercooled liquids. Physical review letters, 85(25):5356, 2000.

[8] Kurt Broderix, Kamal K Bhattacharya, Andrea Cavagna, Annette Zippelius, and Irene Giardina. Energy landscape of a lennard-jones liquid: statistics of stationary points. Physical review letters, 85(25):5360, 2000.

[9] J. P. K. Doye and D. J. Wales. Saddle points and dynamics of lennard-jones clusters, solids, and supercooled liquids. $J$. Chem. Phys., 116:3777-3788, 2002.

[10] Jonathan PK Doye and David J Wales. Comment on 'quasisaddles as relevant points of the potential energy surface in the dynamics of supercooled liquids' j. chem. phys. 116, 10297 (2002). The Journal of chemical physics, 118(11):5263-5264, 2003.

[11] D. J. Wales and J. P. K. Doye. Stationary points and dynamics in high-dimensional systems. J. Chem. Phys., 119:1240912416, 2003.

[12] Juliana Duncan, Qiliang Wu, Keith Promislow, and Graeme Henkelman. Biased gradient squared descent saddle point finding method. The Journal of Chemical Physics, 140(19):-, 2014.

[13] J. Nocedal. Updating quasi-newton matrices with limited storage. Mathematics of Computation, 35:773-782, 1980.

[14] D. Liu and J. Nocedal. On the limited memory bfgs method for large scale optimization. Math. Prog., 45:503-528, 1989.

[15] S. A. Trygubenko and D. J. Wales. A doubly nudged elastic band method for finding transition states. J. Chem. Phys., 120:2082-2094, 2004. 
[16] D. J. Wales. Basins of attraction for stationary-points on a potential-energy surface. J. Chem. Soc. Faraday Trans., 88:653-657, 1992.

[17] D. J. Wales. Locating stationary-points for clusters in cartesian coordinates. J. Chem. Soc. Faraday Trans., 89:1305-1313, 1993.

[18] L. J. Munro and D. J. Wales. Defect migration in crystalline silicon. Phys. Rev. B, 59:3969-3980, 1999.

[19] G. Henkelman and H. Jónsson. A dimer method for finding saddle points on high dimensional potential surfaces using only first derivatives. J. Chem. Phys., 111:7010-7022, 1999.

[20] Y. Kumeda, L. J. Munro, and D. J. Wales. Transition states and rearrangement mechanisms from hybrid eigenvector-following and density functional theory. application to c10h10 and defect migration in crystalline silicon. Chem. Phys. Lett., 341:185194, 2001.

[21] David A. Cox, John Little, and Donal O'Shea. Ideals, Varieties, and Algorithms: An Introduction to Computational Algebraic Geometry and Commutative Algebra, 3/e (Undergraduate Texts in Mathematics). Springer-Verlag New York, Inc., Secaucus, NJ, USA, 2007.

[22] C Ryan Gwaltney, Youdong Lin, Luke D Simoni, and Mark A Stadtherr. Interval methods for nonlinear equation solving applications. Handbook of Granular Computing. Chichester, UK: Wiley, pages 81-96, 2008.

[23] E. L. Allgower and K. Georg. Introduction to Numerical Continuation Methods. John Wiley \& Sons, New York, 1979.

[24] Saeed Khaleghi Rahimian, Farhang Jalali, JD Seader, and RE White. A robust homotopy continuation method for seeking all real roots of unconstrained systems of nonlinear algebraic and transcendental equations. Industrial \& Engineering Chemistry Research, 50(15):8892-8900, 2011.

[25] Saeed Khaleghi Rahimian, Farhang Jalali, JD Seader, and Ralph E White. A new homotopy for seeking all real roots of a nonlinear equation. Computers \& chemical engineering, 35(3):403-411, 2011

[26] A.J. Sommese and C.W. Wampler. The Numerical Solution of Systems of Polynomials Arising in Engineering and Science. World Scientific Publishing, Hackensack, NJ, 2005.

[27] D.J. Bates, J.D. Hauenstein, A.J. Sommese, and C.W. Wampler. Numerically solving polynomial systems with Bertini, volume 25. SIAM, 2013.

[28] Alexander Morgan and Andrew Sommese. Computing all solutions to polynomial systems using homotopy continuation. Applied Mathematics and Computation, 24(2):115-138, 1987.

[29] Jan Verschelde. Algorithm 795: Phcpack: a general-purpose solver for polynomial systems by homotopy continuation. ACM Trans. Math. Soft., 25(2):251-276, 1999.

[30] T L Lee, T Y Li, and C H Tsai. Hom4ps-2.0, a software package for solving polynomial systems by the polyhedral homotopy continuation method. Computing, 83:109-133, 2008.

[31] Tianran Chen, Tsung-Lin Lee, and Tien-Yien Li. Hom4ps-3: A parallel numerical solver for systems of polynomial equations based on polyhedral homotopy continuation methods. In Mathematical Software-ICMS 2014, pages 183-190. Springer, 2014.

[32] Dhagash Mehta. Lattice vs. Continuum: Landau Gauge Fixing and 't Hooft-Polyakov Monopoles. Ph.D. Thesis, The Uni. of Adelaide, Australasian Digital Theses Program, 2009.

[33] Dhagash Mehta. Finding All the Stationary Points of a Potential Energy Landscape via Numerical Polynomial Homotopy Continuation Method. Phys.Rev., E84:025702, 2011.

[34] Dhagash Mehta. Numerical Polynomial Homotopy Continuation Method and String Vacua. Adv.High Energy Phys., 2011:263937, 2011.

[35] Michael Kastner and Dhagash Mehta. Phase Transitions
Detached from Stationary Points of the Energy Landscape Phys.Rev.Lett., 107:160602, 2011.

[36] Markos Maniatis and Dhagash Mehta. Minimizing Higgs Potentials via Numerical Polynomial Homotopy Continuation. Eur.Phys.J.Plus, 127:91, 2012.

[37] Dhagash Mehta, Yang-Hui He, and Jonathan D. Hauenstein Numerical Algebraic Geometry: A New Perspective on String and Gauge Theories. JHEP, 1207:018, 2012.

[38] Ciaran Hughes, Dhagash Mehta, and Jon-Ivar Skullerud. Enumerating Gribov copies on the lattice. Annals Phys., 331:188215, 2013.

[39] Dhagash Mehta, Jonathan D. Hauenstein, and Michael Kastner. Energy-landscape analysis of the two-dimensional nearestneighbor $\phi^{4}$ model. Phys. Rev. E, 85:061103, Jun 2012.

[40] Danny Martinez-Pedrera, Dhagash Mehta, Markus Rummel, and Alexander Westphal. Finding all flux vacua in an explicit example. JHEP, 1306:110, 2013.

[41] Yang-Hui He, Dhagash Mehta, Matthew Niemerg, Markus Rummel, and Alexandru Valeanu. Exploring the Potential Energy Landscape Over a Large Parameter-Space. JHEP, 1307:050, 2013

[42] Dhagash Mehta, Daniel A. Stariolo, and Michael Kastner. Energy landscape of the finite-size spherical three-spin glass model. Phys.Rev., E87(5):052143, 2013.

[43] Brian Greene, David Kagan, Ali Masoumi, Dhagash Mehta, Erick J. Weinberg, et al. Tumbling through a landscape: Evidence of instabilities in high-dimensional moduli spaces. Phys.Rev., D88(2):026005, 2013.

[44] Wenrui Hao, Rafael I. Nepomechie, and Andrew J. Sommese. Completeness of solutions of Bethe's equations. Phys.Rev. E88(5):052113, 2013

[45] Ciaran Hughes, Dhagash Mehta, and David J Wales. An Inversion-Relaxation Approach for Sampling Stationary Points of Spin Model Hamiltonians. J.Chem.Phys., 140:194104, 2014.

[46] A. Abraham C. Grosan. A new approach for solving nonlinear equation systems. IEEE Transactions on Systems Man and Cybernetics - Part A, 38(3):698-714, 2008.

[47] V. Snasel C. Grosan, A. Abraham. Solving polynomial systems using a modified line search approach. J. of Innovative Computing, Information and Control, 8(1):1-10, 2012.

[48] W. Song Y. Wang H.X. Li Z. Cai. Locating multiple optimal solutions of nonlinear equation systems based on multiobjective optimization. IEEE Transactions on Evolutionary Computation, 2014.

[49] J. Wang. Immune genetic algorithm for solving nonlinear equations. Proceedings of the International Conference on Mechatronic Science, Electric Engineering and Computer, pages 2094-2097, 2011.

[50] J. Liu J. Wu, Z. Cui. Using hybrid social emotional optimization algorithm with metropolis rule to solve nonlinear equations. IEEE International Conference on Cognitive Informatics and Cognitive Computing, pages 405-411, 2011.

[51] D. Abdollahi M. Abdollahi, A. Isazadeh. Imperialist competitive algorithm for solving systems of nonlinear equations. Computers and Mathematics with Applications, 65(12):18941908, 2013.

[52] G. Zhao Y. Zhou, J. Liu. Leader glowworm swarm optimization algorithm for solving nonlinear equations systems. Electrical Review, 88(1):101-106, 2012.

[53] Q. Wang Y. Mo, H. Liu. Conjugate direction particle swarm optimization solving systems of nonlinear equations. Computers and Mathematics with Applications, 57(11-12):1877-1882, 2009.

[54] B. Karimi M. Jaberipour, E. Khorram. Particle swarm algorithm for solving systems of nonlinear equations. Computers and Mathematics with Applications, 62(2):566-576, 2011. 
[55] M. G. C. Resende M. J. Hirsch, P. M. Pardalos. Solving system of nonlinears equations with continuous grasp. Nonlinear Analysis: Real World Applications, 10(4):2000-2006, 2009.

[56] M. Mirzaei M. Shams A. Pourrajabian, R. Ebrahimi. Applying genetic algorithms for solving nonlinear algebraic equations. Applied Mathematics and Computation, 219(24):11483-11494, 2013.

[57] P. J. Fleming R. Wang, R. C. Purshouse. Preference-inspired co-evolutionary algorithms for many-objective optimisation. IEEE Transactions on Evolutionary Computation, 17(4):474494, 2013.

[58] S. Effati and A. R. Nazemi. A new method for solving a system of the nonlinear equations. Applied Mathematics and Computation, 168(2):877-894, 2005.

[59] D. J. Wales and J. P. K. Doye. Stationary points and dynamics in high-dimensional systems. Journal of Chem. Phys., 119:12409-12416, December 2003.

[60] Michael Kastner. Phase transitions and configuration space topology. Rev. Mod. Phys., 80(1):167-187, 2008.

[61] Z. Li and H. A. Scheraga. Monte carlo-minimization approach to the multiple-minima problem in protein folding. Proc. Natl. Acad. Sci. USA, 84:6611, 1987.

[62] D. J. Wales and J. P. K. Doye. Global optimization by basin-hopping and the lowest energy structures of lennardjones clusters containing up to 110 atoms. J. Phys. Chem. A, 101:5111, 1997.

[63] D. J. Wales and H. A. Scheraga. Global optimization of clusters, crystals and biomolecules. Science, 285:1368-1372, 1999.

[64] T. V. Bogdan, D. J. Wales, and F. Calvo. Equilibrium thermodynamics from basin-sampling (13 pages). J. Chem. Phys., 124:044102, 2006.

[65] V. A. Sharapov, D. Meluzzi, and V. A. Mandelshtam. Lowtemperature structural transitions: Circumventing the brokenergodicity problem. Phys. Rev. Lett., 98:105701, 2007.

[66] V. A. Sharapov and V. A. Mandelshtam. Solid-solid structural transformations in lennard-jones clusters: Accurate simulations versus the harmonic superposition approximation. J. Phys. Chem. A, 111:10284-10291, 2007.

[67] D. J. Wales. Surveying a complex potential energy landscape: Overcoming broken ergodicity using basin-sampling. Chem. Phys. Lett., 584(0):1 - 9, 2013.

[68] D.J. Wales. Discrete path sampling. Mol. Phys, 100:3285-3306, 2002.

[69] D. J. Wales. Some further applications of discrete path sampling to cluster isomerization. Mol. Phys., 102:891-908, 2004.

[70] D. J. Wales. Energy landscapes: Calculating pathways and rates. Int. Rev. Phys. Chem., 25:237-282, 2006.

[71] G. C. Boulougouis and D. N. Theodorou. Dynamical integration of a markovian web: a first passage time approach. $J$. Chem. Phys., 127:084903, 2007.

[72] L. Xu and G. Henkelman. Adaptive kinetic monte carlo for first-principles accelerated dynamics (9 pages). J. Chem. Phys., 129:114104, 2008.

[73] Roberto Franzosi, Marco Pettini, and Lionel Spinelli. Topology and phase transitions: Paradigmatic evidence. Phys. Rev. Lett., 84(13):2774-2777, Mar 2000.

[74] D. Mehta, J. D. Hauenstein, and M. Kastner. Energy-landscape analysis of the two-dimensional nearest-neighbor $\phi^{4}$ model. Phys. Rev. E, 85:061103, 2012.

[75] Joseph John Thomson. Xxiv. on the structure of the atom: an investigation of the stability and periods of oscillation of a number of corpuscles arranged at equal intervals around the circumference of a circle; with application of the results to the theory of atomic structure. The London, Edinburgh, and Dublin
Philosophical Magazine and Journal of Science, 7(39):237265, 1904

[76] Nikolay N Andreev. An extremal property of the icosahedron. East J. Approx, 2(4):459-462, 1996.

[77] Richard Evan Schwartz. The 5 electron case of thomson's problem. arXiv preprint arXiv:1001.3702 2010.

[78] Steve Smale. Mathematical problems for the next century. The Mathematical Intelligencer, 20(2):7-15, 1998.

[79] Diego Armentano, Carlos Beltrán, and Michael Shub. Minimizing the discrete logarithmic energy on the sphere: The role of random polynomials. Transactions of the American Mathematical Society, 363(6):2955-2965, 2011.

[80] J. E. Jones and A. E. Ingham. On the calculation of certain crystal potential constants, and on the cubic crystal of least potential energy. Proc. R. Soc. A, 107:636-653, 1925.

[81] J. P. K. Doye and C. P. Massen. Characterizing the network topology of the energy landscapes of atomic clusters. J. Chem. Phys., 122(8):084105, February 2005.

[82] Dhagash Mehta, Tianran Chen, Jonathan D Hauenstein, and David J Wales. Communication: Newton homotopies for sampling stationary points of potential energy landscapes. The Journal of Chemical Physics, 141(12):121104, 2014

[83] Philip M. Morse. Diatomic molecules according to the wave mechanics. ii. vibrational levels. Phys. Rev., 34:57-64, Jul 1929.

[84] J Michael Kosterlitz and D James Thouless. Ordering, metastability and phase transitions in two-dimensional systems. Journal of Physics C: Solid State Physics, 6(7):1181, 1973.

[85] Juan A Acebrón, Luis L Bonilla, Conrad J Pérez Vicente, Félix Ritort, and Renato Spigler. The kuramoto model: A simple paradigm for synchronization phenomena. Reviews of modern physics, 77(1):137, 2005.

[86] Axel Maas. Describing gauge bosons at zero and finite temperature. Phys.Rept., 524:203-300, 2013.

[87] Dhagash Mehta and Michael Kastner. Stationary point analysis of the one-dimensional lattice Landau gauge fixing functional, aka random phase XY Hamiltonian. Annals Phys., 326:14251440, 2011.

[88] Lorenz von Smekal, Dhagash Mehta, Andre Sternbeck, and Anthony G. Williams. Modified Lattice Landau Gauge. PoS, LAT2007:382, 2007.

[89] Lorenz von Smekal, Alexander Jorkowski, Dhagash Mehta, and Andre Sternbeck. Lattice Landau gauge via Stereographic Projection. PoS, CONFINEMENT8:048, 2008.

[90] Rachele Nerattini, Michael Kastner, Dhagash Mehta, and Lapo Casetti. Exploring the energy landscape of XY models. Phys.Rev., E87(3):032140, 2013.

[91] Dhagash Mehta and Mario Schröck. Enumerating copies in the first gribov region on the lattice in up to four dimensions. 2014.

[92] Dhagash Mehta, Andre Sternbeck, Lorenz von Smekal, and Anthony G Williams. Lattice Landau Gauge and Algebraic Geometry. PoS, QCD-TNT09:025, 2009.

[93] Dhagash Mehta, Noah Daleo, Florian Dörfler, and Jonathan D Hauenstein. Algebraic geometrization of the kuramoto model: Equilibria and stability analysis. arXiv preprint arXiv:1412.0666. 2014.

[94] N. Akino and J. M. Kosterlitz. Domain wall renormalization group study of the $x y$ model with quenched random phase shifts. Phys. Rev. B, 66(5):054536, Aug 2002.

[95] Christian Kanzow Francisco Facchinei. Generalized nash equilibrium problems. Annals of Operations Research, 175(1):177211,2010

[96] B. Sturmfels. Solving systems of polynomial equations. CBMS Regional Conference Series in Math, American Mathematical Society, Providence, RI, 97, 2002. 
[97] Martin L. Demaine Erik D. Demaine. Jigsaw puzzles, edge matching, and polyomino packing: Connections and complexity. Graphs and Combinatorics, 23:195-208, 2007.

[98] R. Basri S.Z. Kovalsky, D. Glasner. A global approach for solving edge-matching puzzles. arXiv.org cs arXiv:1409.5957 2014. 